Supplement of SOIL, 8, 85-97, 2022

https://doi.org/10.5194/soil-8-85-2022-supplement

(c) Author(s) 2022. CC BY 4.0 License.

(c) (i)

Supplement of

\title{
An underground, wireless, open-source, low-cost system for monitoring oxygen, temperature, and soil moisture
}

\section{Elad Levintal et al.}

Correspondence to: Elad Levintal (elevintal@ucdavis.edu)

The copyright of individual parts of the supplement might differ from the article licence. 


\section{General notes:}

- The information provided below gives detailed instructions for the LoRa-WUSN system developed and tested in this study. For other applications, we anticipate that the user will likely have to make small adjustments depending on the project goals and any differences in electronics (e.g., sensor types, hardware versions and/or suppliers). This is a standard notation when using open-source hardware, as also stated in previous studies aimed at utilizing this type of hardware in the research world (Bastviken et al., 2020).

- One of the open-source hardware community fundamental principles is sharing knowledge and information. Therefore, there are many online resources (webinars, tutorials, movies, product pages, etc.) users can make use of that start at the beginner's level (e.g., how to solder). We highly recommend using them if needed.

- Please consult with your institute's safety representative regarding soldering and any other related issue that can pose a fire or safety risk.

- Hardware and software are not necessarily free to use for any purpose, even if available online. Licensing is a complex and evolving field in open-source hardware. In general, the Arduino IDE and related libraries are under the GNU Lesser General Public License (LGPL) (Chan et al., 2020). It is also important to recognize the contributor of each code section, library, or any other professional information if used or duplicated within the system (e.g., see the comments within the code in section 2).

- The authors are glad to answer and help in issues related to this study. We are also interested in learning and improving. Therefore, communication with the end-user will be valued.

\section{Hardware}




\section{General hardware notes:}

- The hardware below is based on the component list provided in Table 1 in the main text. Additional miscellaneous needed equipment include: Plasti Dip or epoxy (e.g., Loctite 9460 Hysol Epoxy Adhesive) to waterproof the hardware, a hydrophobic membrane (PTFE type, e.g., PTFE gasket sheet, model 95665K92 from McMaster), spacers (e.g., nylon type), heat-shrink tubing, $\sim 5 \mathrm{~m}$ of a 2-wire cable to extend the oxygen sensors' wires, and general soldering equipment.

- We recommend adding heat-shrink tubing to isolate wires when soldering wires to headers - examples are shown in the figures below.

- Each 5TM sensor (soil moisture sensor) should have a unique address due to the SDI-12 communication protocol; instructions can be found in the sensor manual or other online sources (e.g., the example code named b_address_change in the Arduino-SDI-12 library). Programming each sensor's address should be done before connecting all the four sensors together because only one sensor can be connected to a microcontroller when defining its specific address. The four addresses used here are: ' 1 ', '2', '3', and '4'.

\subsection{Assembling the underground node - step-by-step instructions}

1.1.1. Solder the header kit (Header Kit for Feather - 12-pin and 16-pin Female Header Set) to the Adafruit Feather M0 with RFM95 LoRa Radio, hereafter called LoRa-Feather. We note that there are excellent tutorials for learning how to solder headers (e.g., https://learn.adafruit.com/how-to-solder-headers). 
1.1.2. Solder the uFL SMT antenna connector the LoRa-Feather (Fig. S1, yellow arrow), and then connect the antenna cable to it (for more detailed instructions, see https://learn.adafruit.com/adafruit-feather-m0-radio-with-lora-radio-module/antenna-options).

1.1.3. Solder a short wire to the signal port in the power relay (Adafruit Non-Latching Mini Relay FeatherWing) (Fig. S2, yellow arrow), leave the other end free.

1.1.4. Solder the short header kit (Short Feather Male Headers - 12-pin and 16-pin Male Header Set) to the power relay (Adafruit Non-Latching Mini Relay FeatherWing), and then mount it on the LoRa-Feather (Fig. S3, yellow arrow).

1.1.5. Solder the free end of the relay wire (step 1.1.3) to pin \#13 (Fig. S2, yellow circle). For each soldering of wire to pin, remember to add a shrink to the wire before you solder.

1.1.6. Solder a red wire to the $3 \mathrm{~V}$ port (Fig. S2, blue circle) and connect the other end to the COM port (Fig. S2, blue arrow).

1.1.7. Solder four short ( $10 \mathrm{~cm})$ wires to the ADS1015 (the analog-to-digital converter (ADC)) pins named VCC (red), GND (black), SCL (white), and SDA (green) (Fig. S2, green arrow). Note that wire colors are not mandatory, but it is recommended to follow the same logic for all components (e.g., red wire for voltage).

\subsubsection{Preparations of the oxygen sensors}

Cut a $50 \mathrm{ml}$ tube to fit the length of the KE-25 oxygen sensor (Fig. S4, yellow arrow).

Drill a hole on the blue tube cup to insert the black and red sensor wires (Fig. S4). 
Connect the black and red sensor's wires to an extension cable (e.g., via soldering) and use a shrink or any other preferred method to ensure the connections will work once installed underground (Fig. S4, yellow circle).

Connect a hydrophobic membrane (PTFE type) to the bottom of the $50 \mathrm{ml}$ tube. Use Plasti Dip (3-4 layers) or epoxy to seal the connection so it will be waterproof (Fig. S5a). Use the same material to seal the wires hole on the tube cup (Fig. S5b).

Drill a hole in the underground node's plastic box and insert the extension cable through the hole (Fig. S6, blue arrow).

Repeat steps 1.1.8.1 to 1.1.8.5 for each oxygen sensor.

\subsubsection{Preparations of the soil moisture sensors}

Drill holes in the underground node's plastic box and insert the four stereo jack to pigtail probe adapters through the holes (Fig. S6, purple arrow).

Solder each set of four wires together (Fig. S6, purple circles).

Connect the four 5TM to the stereo connections outside the plastic box.

Connect a wire (via soldering) between the four data wires (Fig. S6, purple circle named Data wires) and pin \#5 on the relay.

1.1.10. Drill a hole for the antenna and connect it (Fig. S6, green arrow).

1.1.11. Solder the red wire of oxygen sensor \#1 to the A0 pin on the ADS1015 board, red wire of sensor \#2 to A1, and so on (Fig. S2, green circle). 
1.1.12. Combine all ground cables (i.e., the ADS1015 GND black wire, the four KE-25 black wires, and the four 5TM bare wires) via soldering or any other preferred connector, then solder them to the GND pin on the relay.

1.1.13. Combine the ADS1015 and 5TM power cables (i.e., the ADS1015 VDD red wire and the four 5TM brown wires) via soldering or any other preferred connector, then connect them to the NO port on the relay.

1.1.14. Solder the other white and green ends of the ADS1015 wires to the SCL and SDA pins on the relay, respectively (Fig. S6, yellow arrow).

1.1.15. Mount the ADS1015 on the relay. This can be done using spacers (Fig. 3 blue arrow) or any other preferred method.

1.1.16. Check that all the soldered connections are good and with shrinks, and then connect the $2200 \mathrm{mAh}$ to the LoRa-Feather (Fig. S1, blue arrow).

1.1.17. The following steps for the underground node should only be done after the system was validated and worked as desired (i.e., after uploading the code and testing it, see section 2 below). Make sure to label each sensor; for example, label KE-25 \#1 on the KE-25 connected to A0 on the ADS1015, KE-25 \#2 on the KE-25 connected to A1, and so on.

1.1.18. Mount all components onto the plastic box using epoxy or any other preferred method.

1.1.19. Coat all electronics and holes to prevent humidity and water damage (Fig. S7), including the four 5TM stereo connections outside the box. Make sure not to coat the reset button and the micro USB port (Fig. S7b, yellow and blue arrows, respectively).

1.1.20. The final field setting of the underground node is shown in Fig. S8. 


\subsection{Assembling the underground node - step-by-step instructions}

\subsubsection{Prepare another LoRa-Feather (steps 1.1.1 and 1.1.2).}

1.2.2. Solder the short header kit (Short Feather Male Headers - 12-pin and 16-pin Male Header Set) to the Adalogger FeatherWing, and then mount it on the LoRa-Feather.

1.2.3. Insert the CR1220 battery on the RTC socket (Fig. S9, yellow arrow).

1.2.4. Insert the MicroSD memory card into the MicroSD socket (Fig. S9, blue arrow).

1.2.5. Drill a hole in the aboveground hub's plastic box for the antenna and connect it (Fig. S9, green arrow).

1.2.6. The maximum LoRa-Feather input voltage is $5 \mathrm{~V}$. Therefore, connecting a $12 \mathrm{~V}$ source needs adjustments. Here, we present two options:

Drill a hole in the plastic box and insert the $12 \mathrm{~V}$ battery cable through the hole (Fig. S9, purple circle (a)), then connect the DC/DC Step-Down (Buck) Converter from one end to the $12 \mathrm{~V}$ input cable and the other end to the two power wires of a micro USB cable (Fig. S9, purple circle (b)). Ensure which end of the DC/DC is connected to the $12 \mathrm{~V}$ by following the orientation shown in Fig. $\mathbf{S 9}$ or as described on the product web page.

Another option is to use a DC/DC converter from $12 \mathrm{~V}$ to $5 \mathrm{~V}$ micro USB (many online options, such as Digi-Key or Amazon). In this case, the $12 \mathrm{~V}$ end will be connected to a $12 \mathrm{~V}$ battery and the $5 \mathrm{~V}$ micro USB to the micro USB port in the LoRa-Feather.

1.2.7. Connect the $1200 \mathrm{mAh}$ to the LoRa-Feather (Fig. S9, purple arrow).

1.2.8. Mount all components onto the plastic box using epoxy or any other preferred method. 
1.2.9. The final field setting of the aboveground hub is shown in Fig. S9.

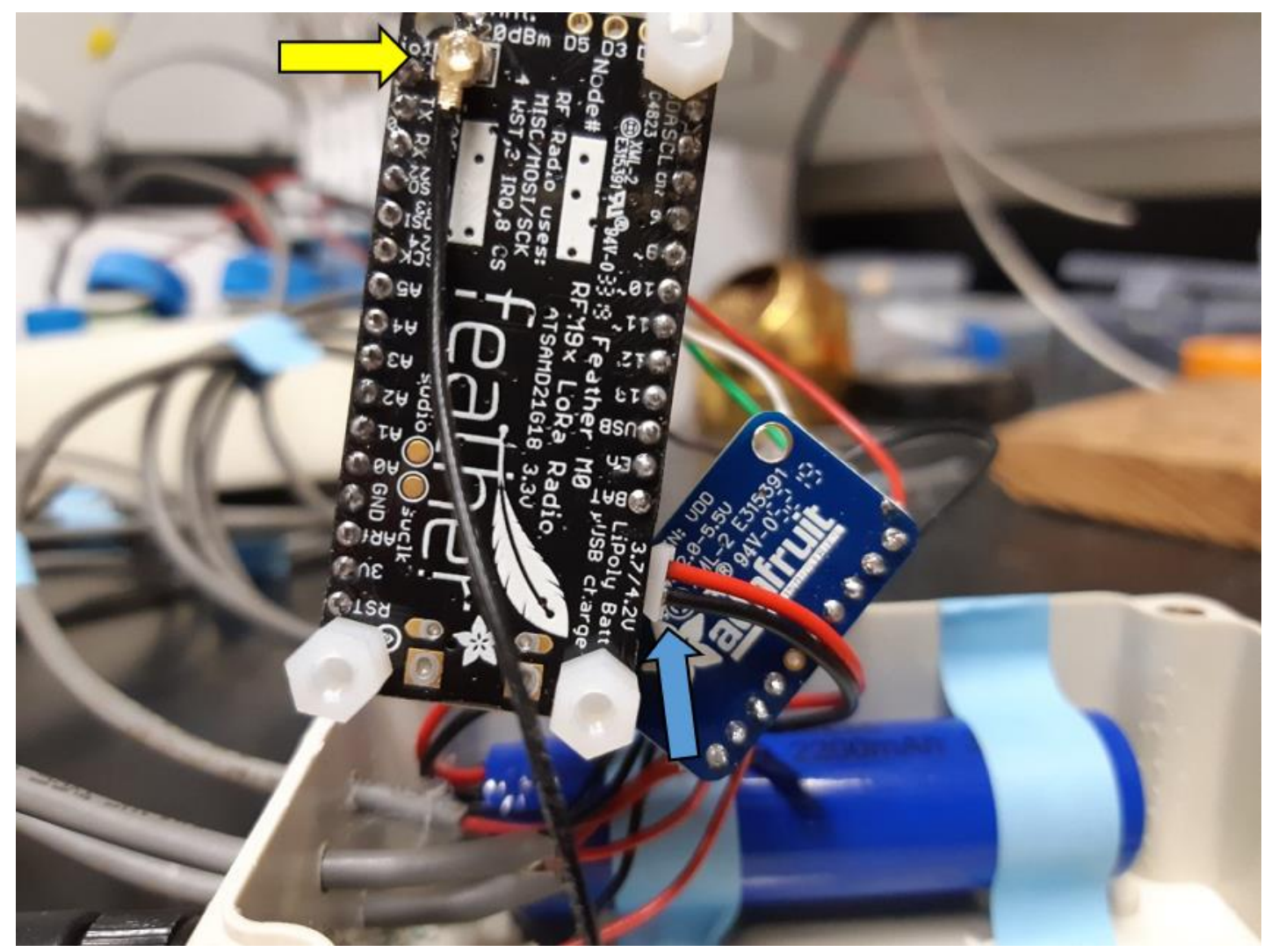

Fig. S1. Bottom view of the LoRa-Feather. The yellow arrow marks the uFL SMT antenna connector (step 1.1.2). 


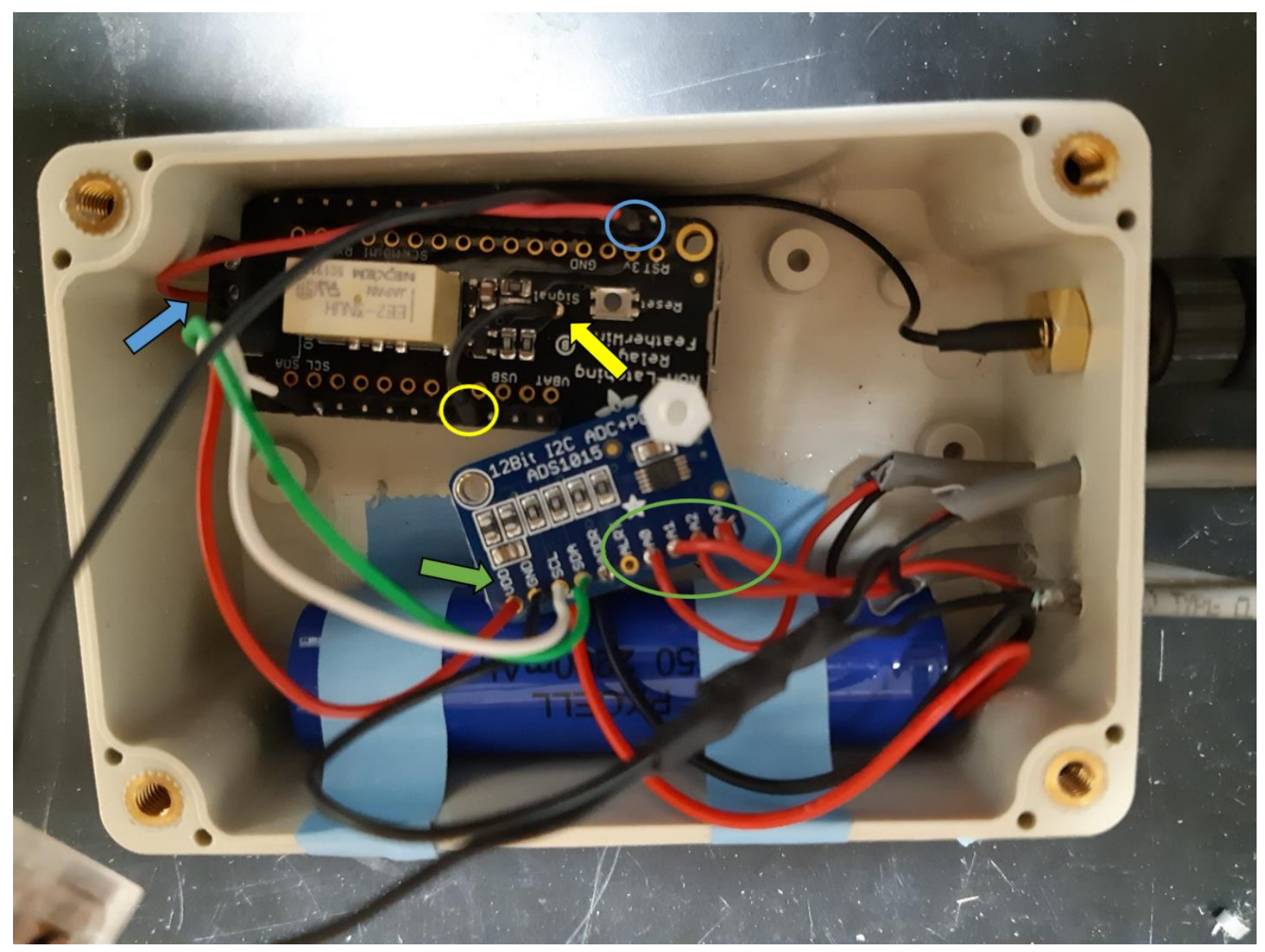

Fig. S2. Underground node overview. The yellow arrow and yellow circle mark the two connections (steps 1.1.3 and 1.1.5, respectively). Blue arrow and blue circle mark the two voltage connection ports between the LoRa-Feather and the relay (step 1.1.6). Green arrow and green circle mark the ADS1015 connection ports (steps 1.1.7 and 1.1.11, respectively). 


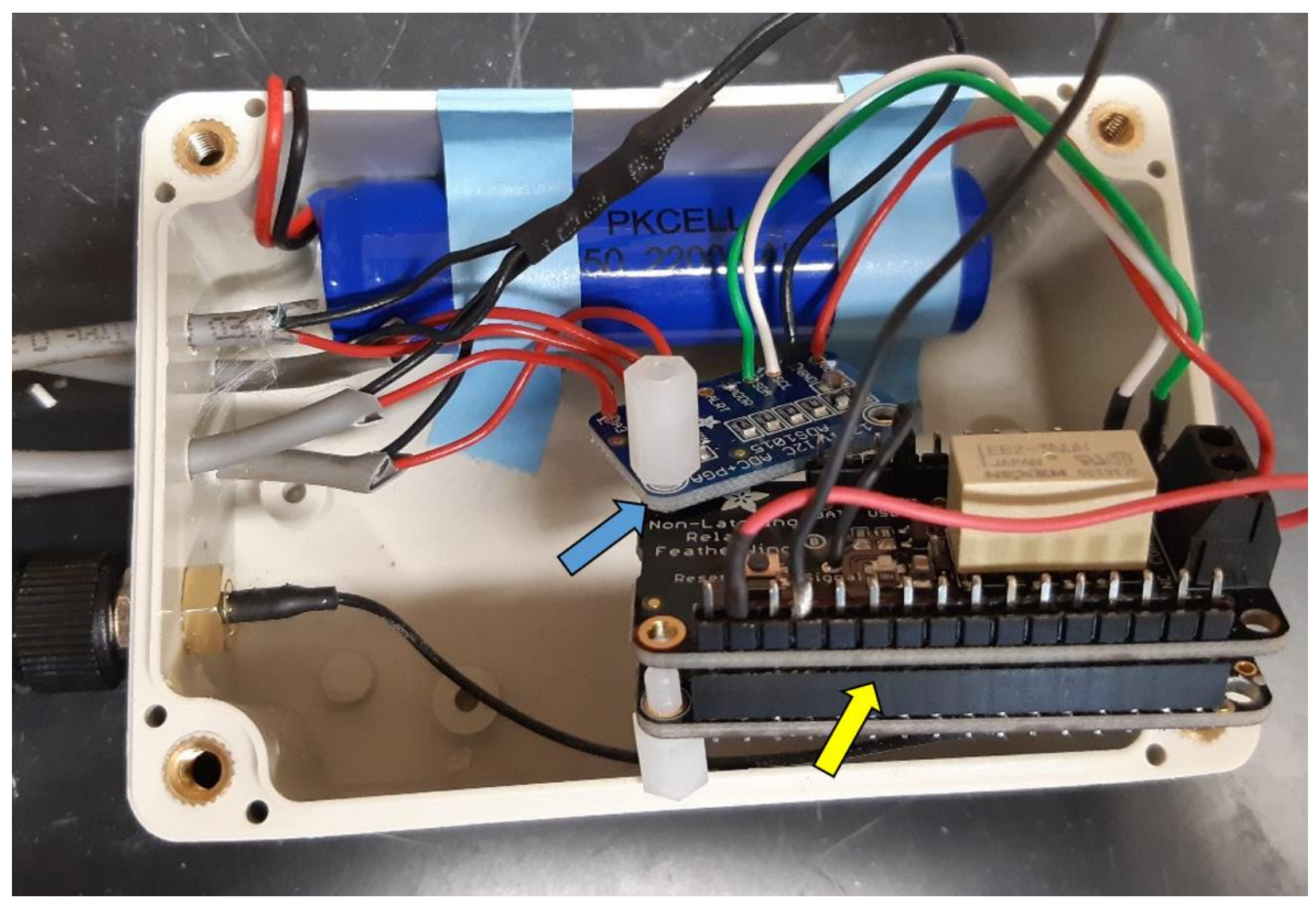

Fig. S3. Underground node overview. The yellow arrow marks the mounting area of the power relay on the LoRa Feather (step 1.1.4). 


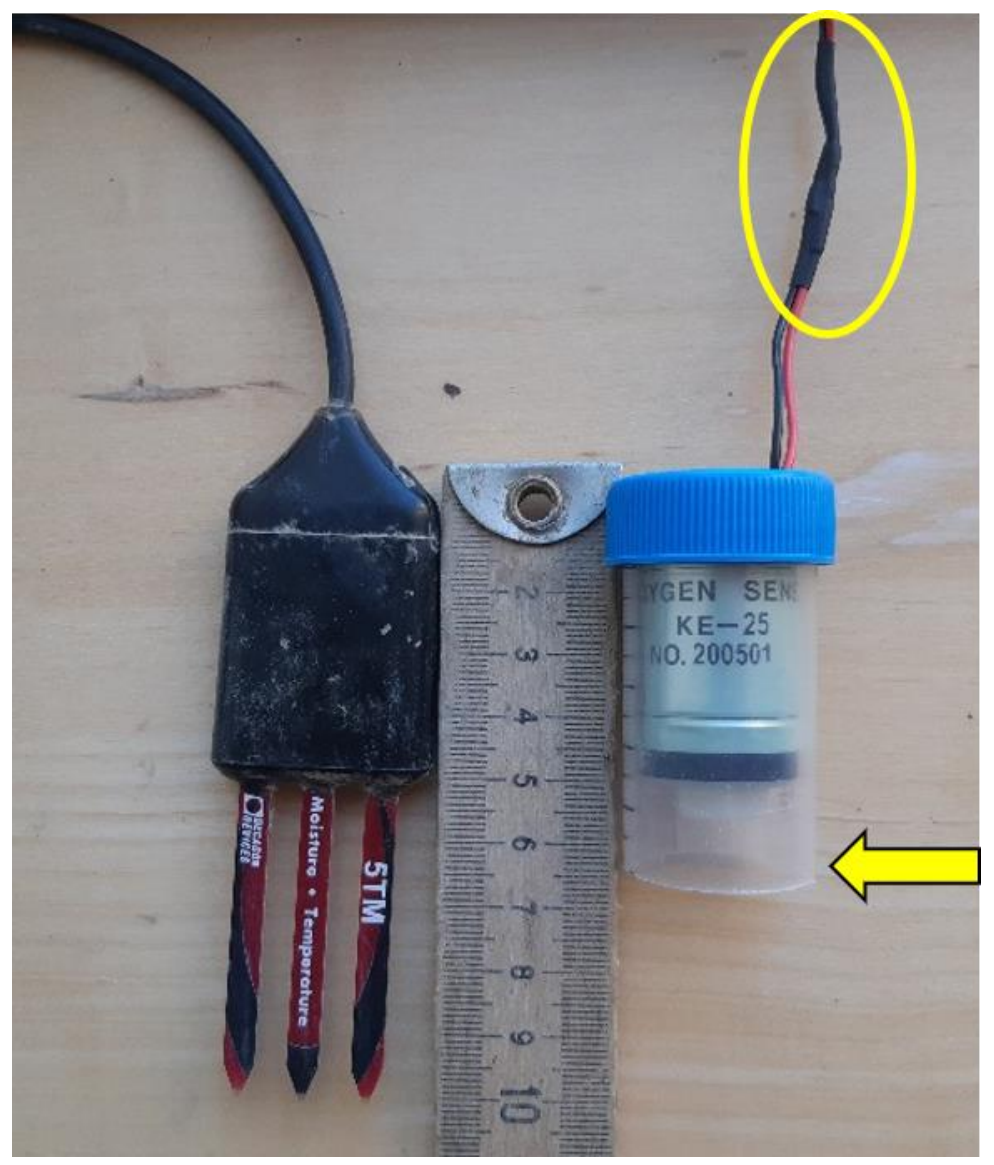

Fig. S4. Sensors overview. The yellow arrow and yellow circle mark the needed tube length (in $\mathrm{cm}$ ) and wire connections (steps 1.1.8.1 and 1.1.8.3, respectively). 

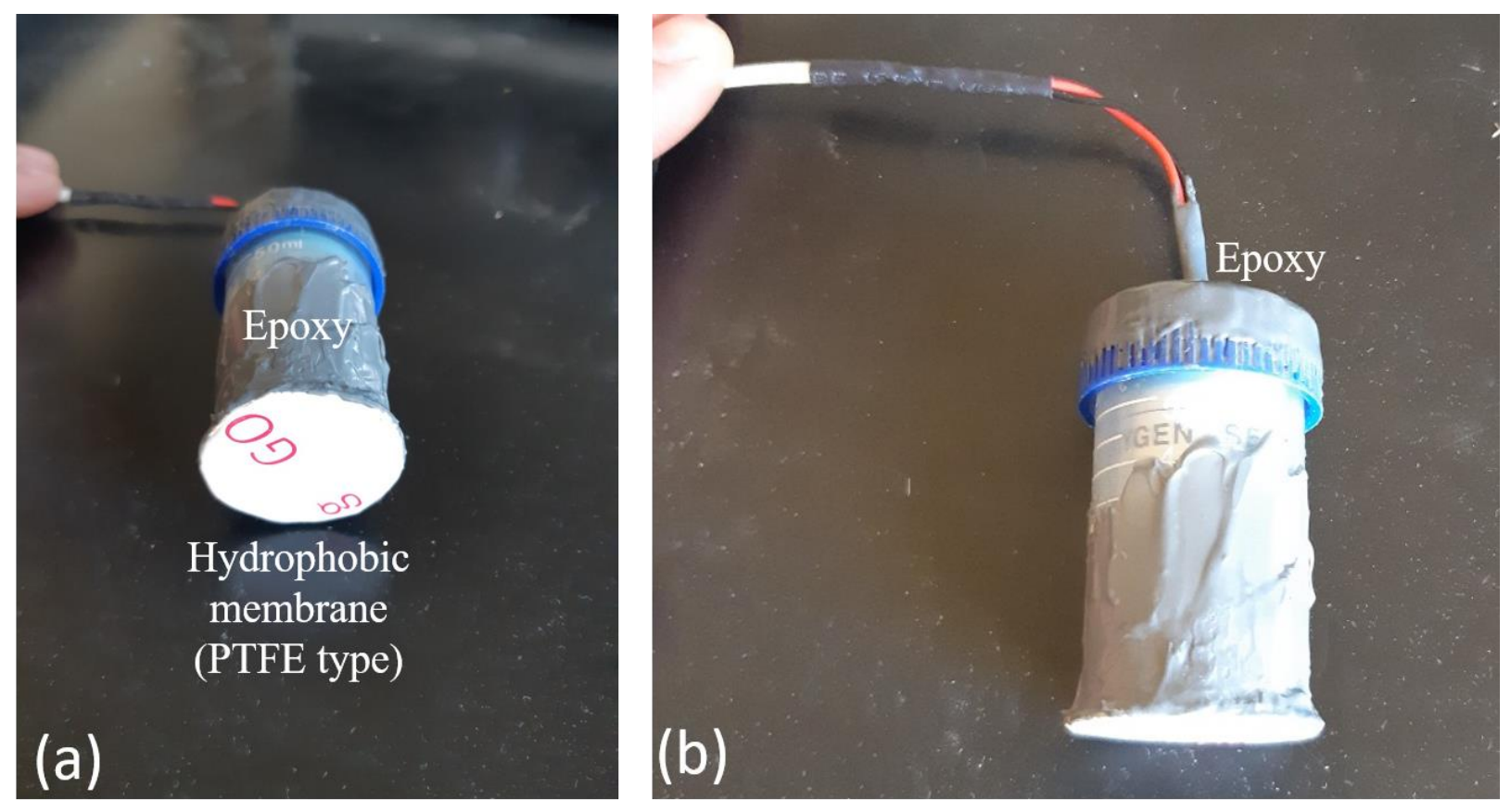

Fig. S5. Example for oxygen sensor waterproofing using a PTFE membrane and epoxy glue (step 1.1.8.4). 


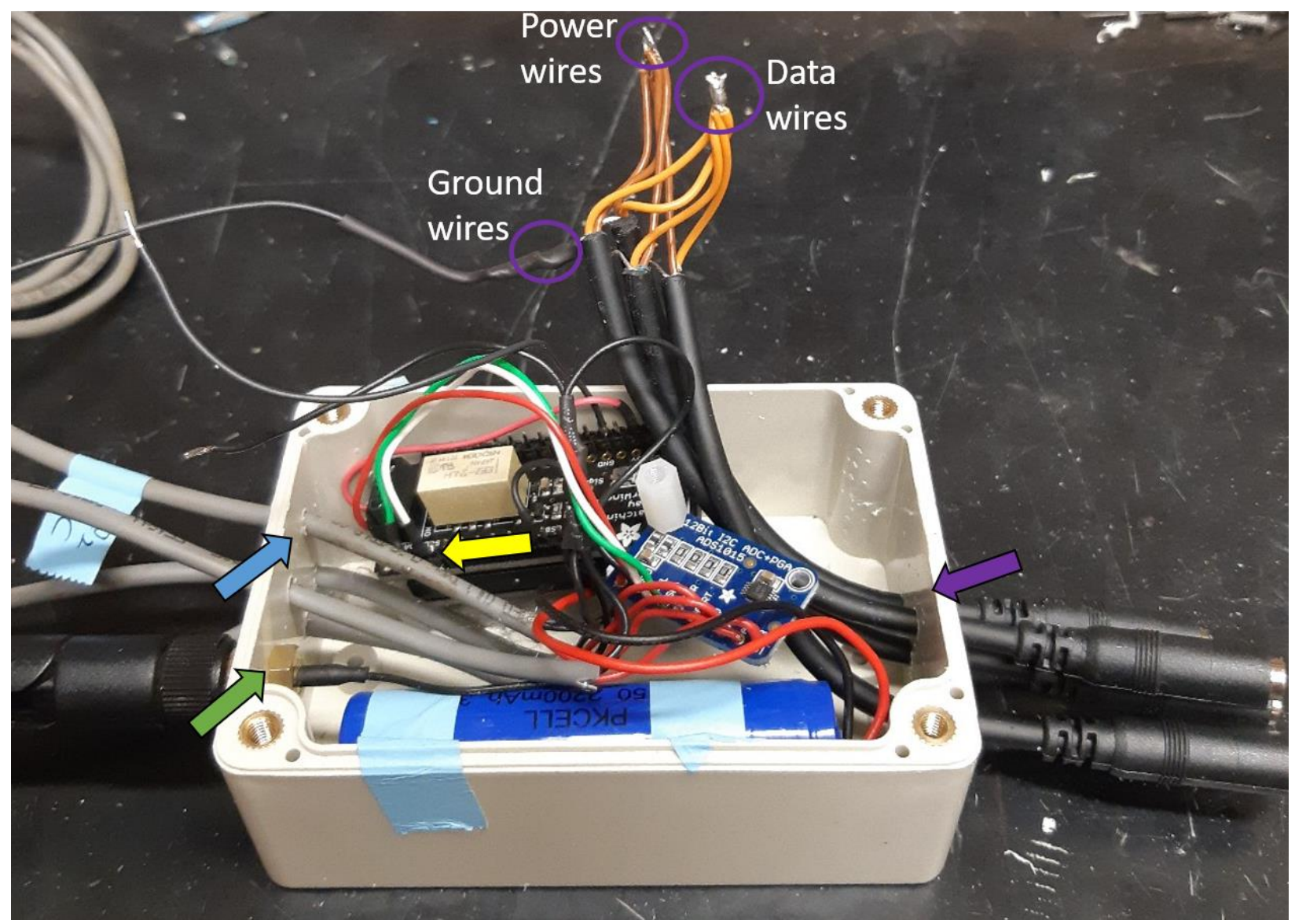

Fig. S6. Underground node overview. The yellow arrow marks the two SCL and SDA pins (step 1.1.14). The blue arrow marks one of the four oxygen cable holes (step 1.1.8.5). The green arrow marks the antenna connection (step 1.1.10). Purple arrow marks the four stereo jack to pigtail probe adapter cable holes (step 1.1.9.1). Purple circles mark the three groups of soldered wires (step 1.1.9.2). 

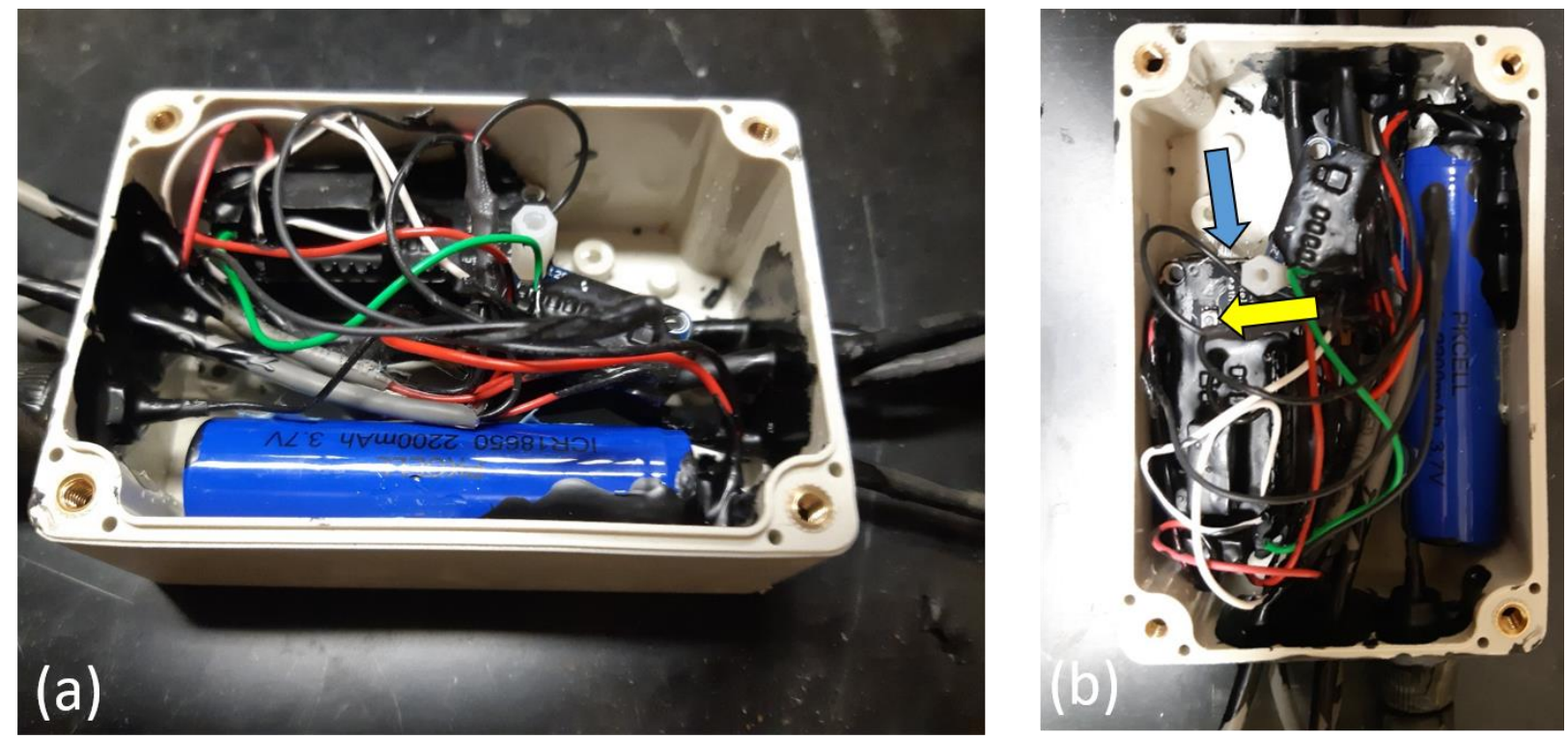

Fig. S7. Overview of the underground node coating using Plasti Dip. Yellow and blue arrows mark the areas that should stay accessible and should not be coated (step 1.1.18). 


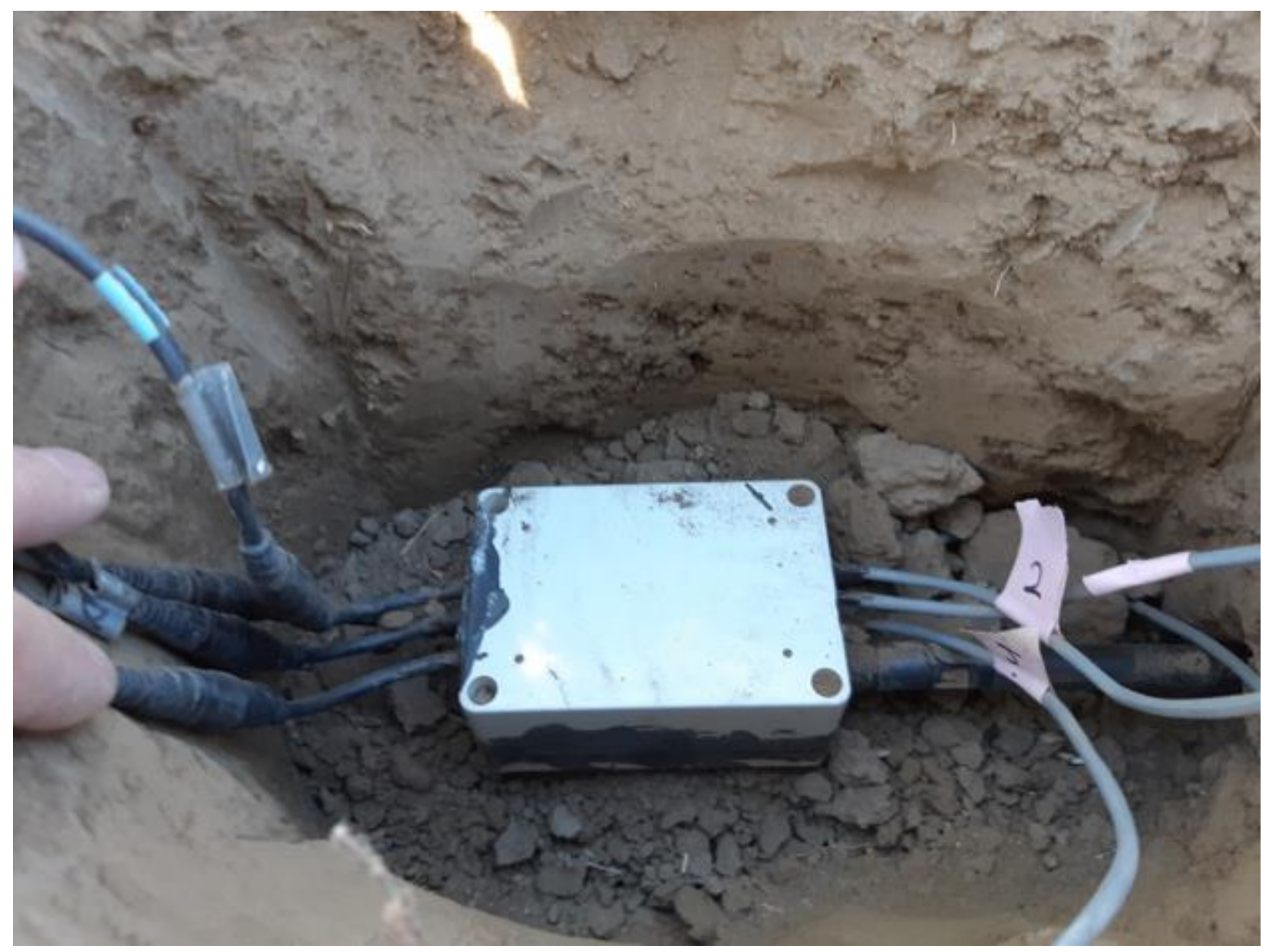

Fig. S8. Field installation of the underground node. 


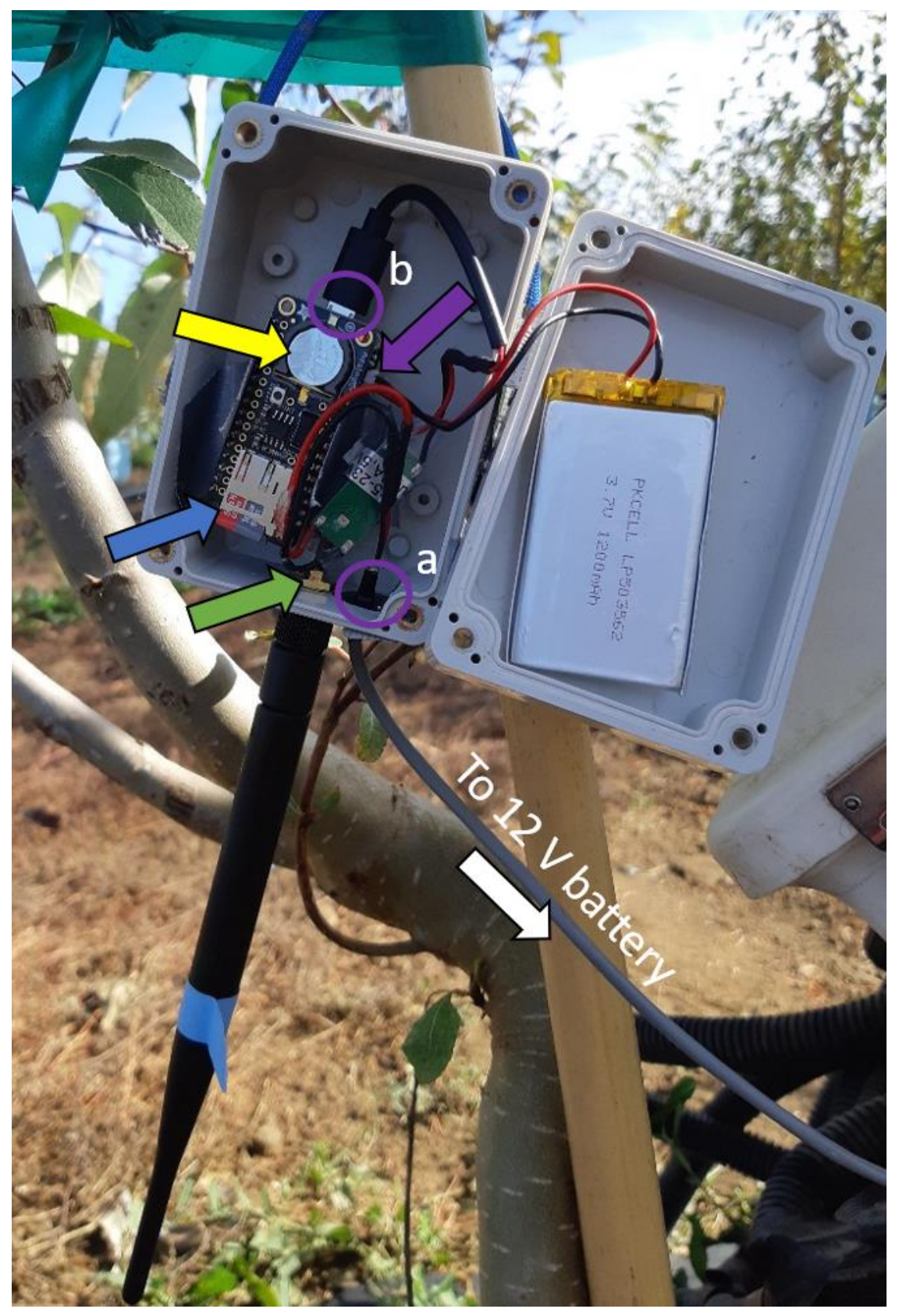

Fig. S9. Aboveground hub overview. The yellow arrow marks the CR1220 location (step 1.2.3).

The blue arrow marks the MicroSD location (step 1.2.4). The green arrow marks the antenna connection (step 1.2.5). Purple arrow marks the 1200 mAh battery connection (step 1.2.7). Purple circles mark connections of the DC/DC Step-Down (Buck) Converter (step 1.2.6.1). 


\section{Software}

The codes for the underground node and the aboveground hub can be found in a GitHub repository at the following link https://github.com/levintal/LoRaSystemForSoils. The code contains comments to explain each code section's purpose and provides the end-user with the flexibility to adjust the system according to specific needs. For more general information and guidelines about open-source hardware programming, one can find numerous online sources and tutorials (as also noted above regarding the hardware). 


\section{Power consumption test}

Fig. S10 and S11 present results from a power consumption test conducted in the lab using the same setting as in the field with the addition of the INA260 (Adafruit, USA) to measure the current at 100-millisecond intervals. Additional results are provided in the main article (Table 2). 


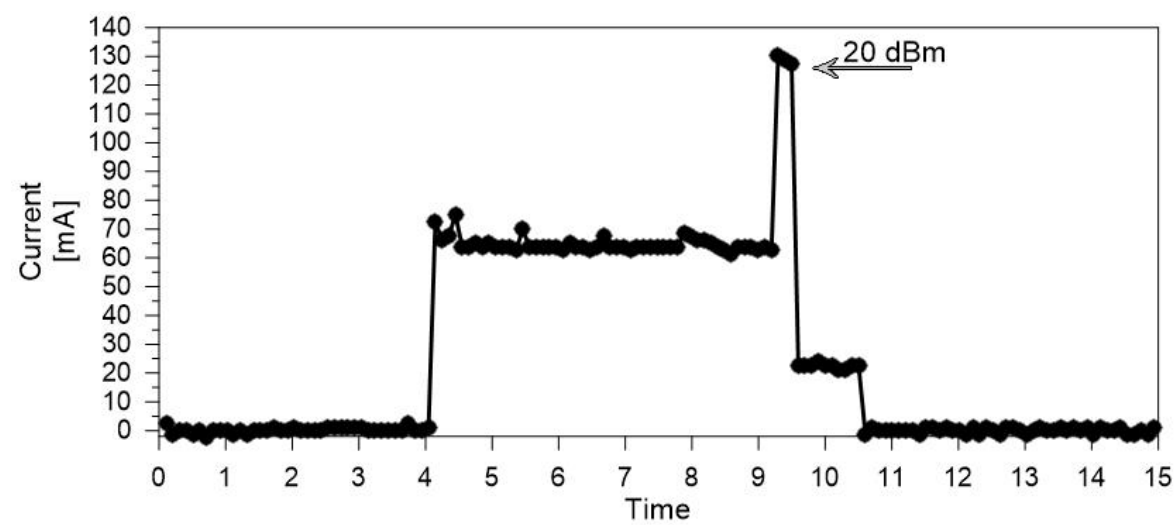

[s]

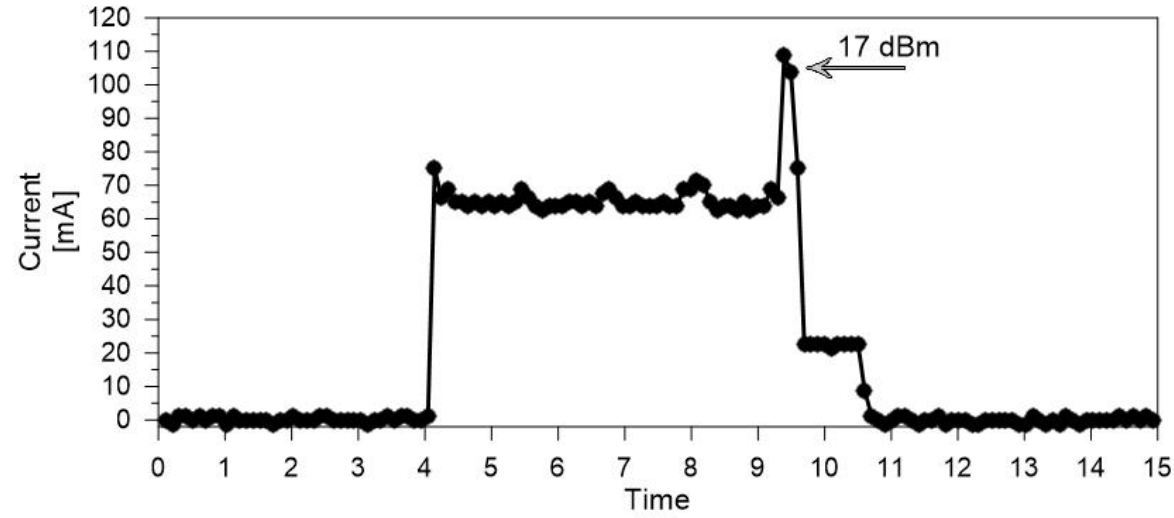

[s]

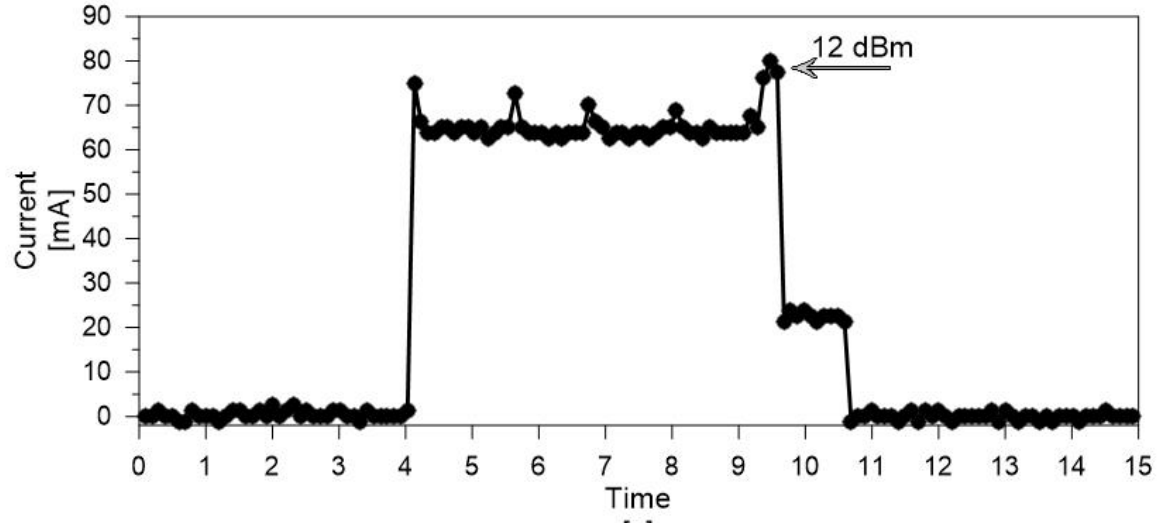

[s]

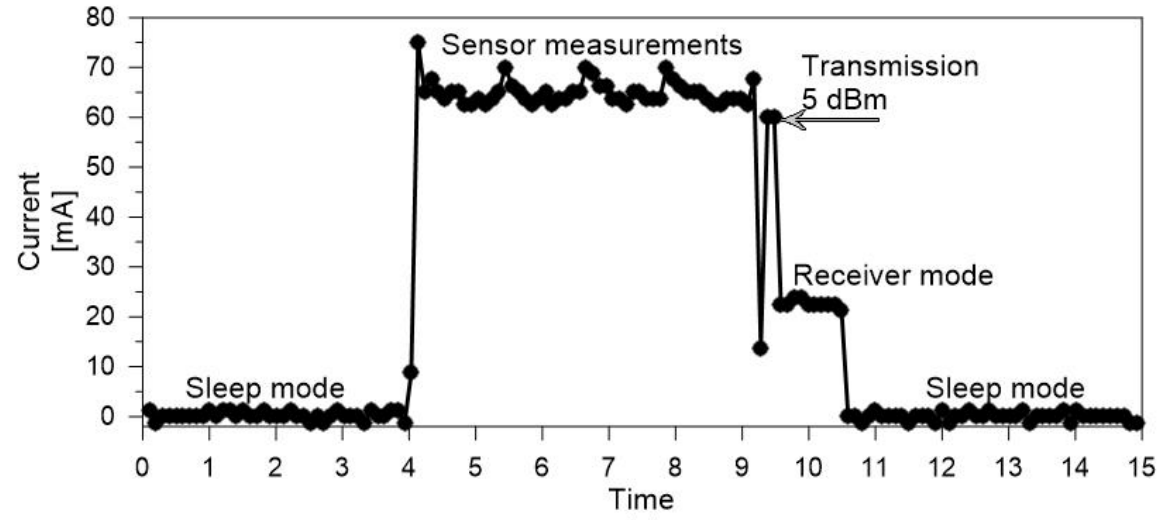

[s] 
Fig. S10. Power consumption during one underground node cycle (sleep mode, sensor measurements, transmission, and receiver mode) for four different transmission levels $(5,12,17$, and $20 \mathrm{dBm})$. 


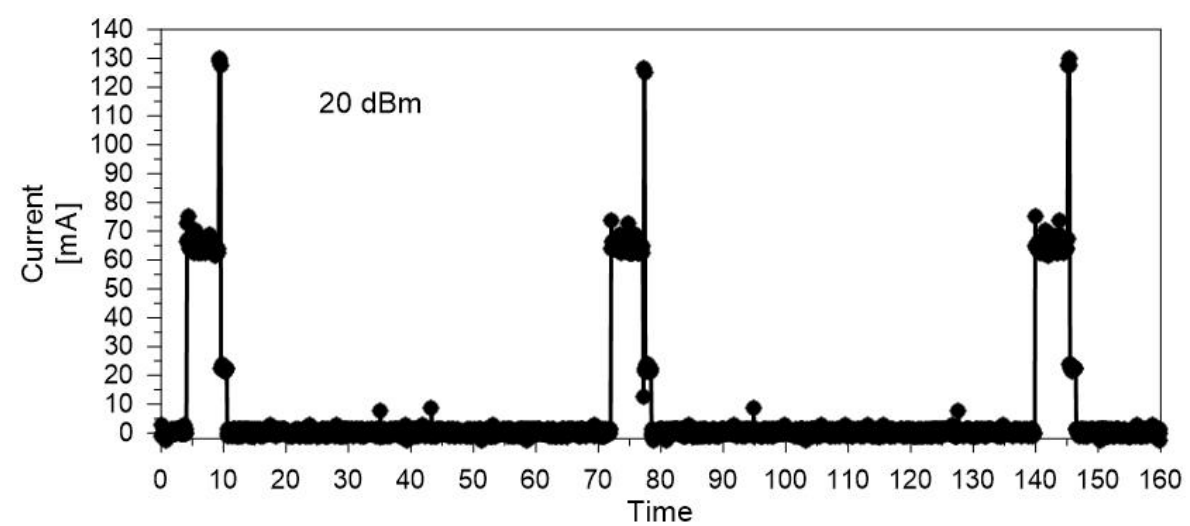

[s]

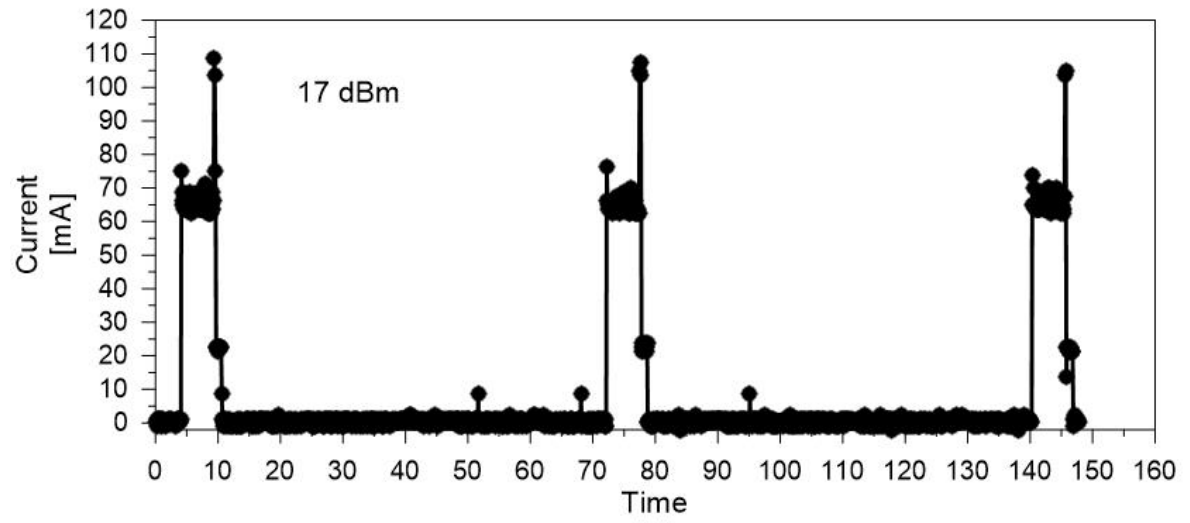

[s]

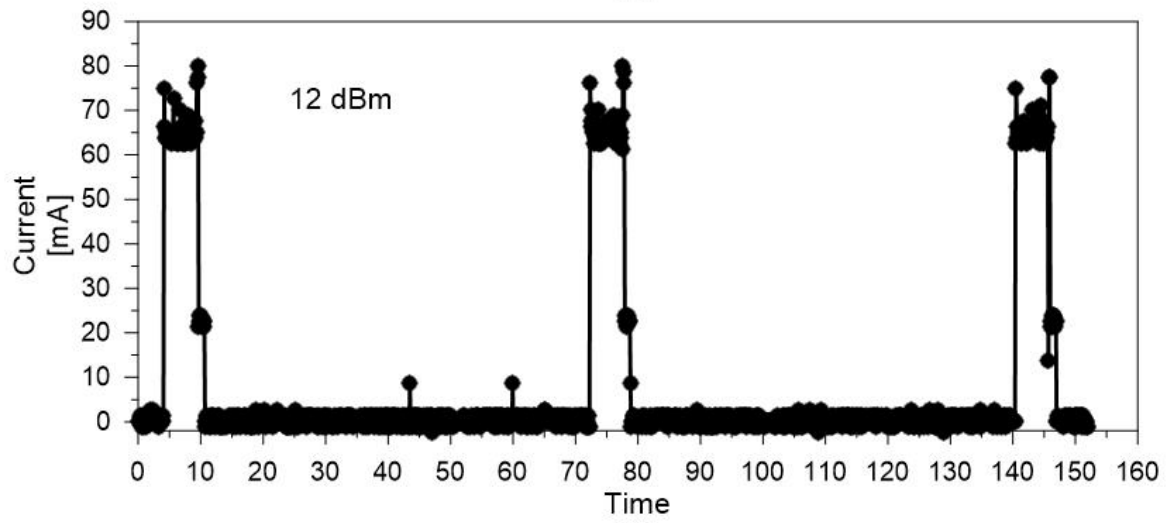

[s]

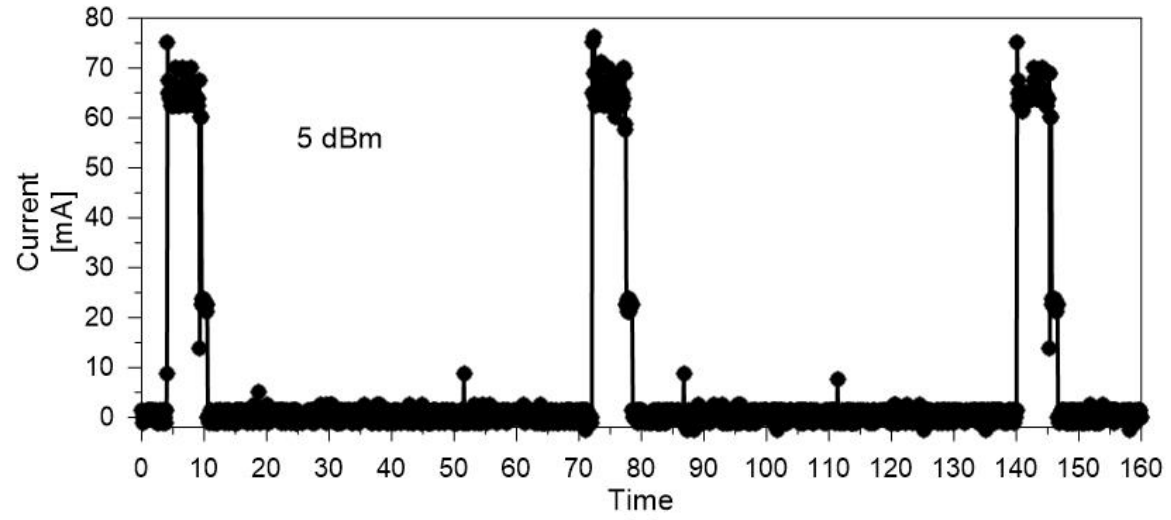

[s] 
Fig. S11. Power consumption during three consecutive underground node cycles (sleep mode, sensor measurements, transmission, and receiver mode) for four different transmission levels (5, $12,17$, and $20 \mathrm{dBm})$. 